\title{
Surface Properties of Alginate/Chitosan Biofilm for Wound Healing Application
}

\author{
M.I. IDRIS ${ }^{1, a *}$, M.F. ADZHARI ${ }^{1, b}$, S.N. ABD. BAKIL ${ }^{1, c}$, T.C. LEE ${ }^{2, d}$, \\ M.A. SELIMIN ${ }^{2, \mathrm{e}}$ and H.Z. ABDULLAH ${ }^{1, \mathrm{f}}$ \\ 1Department of Manufacturing Engineering, Faculty of Mechanical and Manufacturing Engineering, \\ Universiti Tun Hussein Onn Malaysia, 86400 Parit Raja, Batu Pahat, Johor, Malaysia. \\ ${ }^{2}$ Department of Manufacturing and Operating Management, Faculty of Technology Management \\ and Business, Universiti Tun Hussein Onn Malaysia, 86400 Parit Raja, Batu Pahat, Johor, \\ Malaysia.

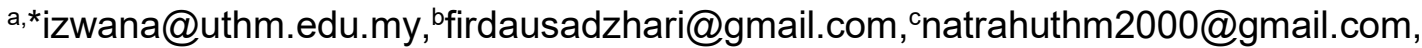

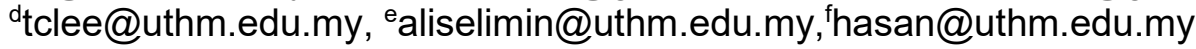

Keywords: Alginate; Chitosan; Biopolymer; Surface properties; Wound healing

\begin{abstract}
This work focuses on the fabrication of film based on natural biopolymers for wound healing application. Alginate and chitosan were choosen because of their oustanding properties such as biocompatible, hydrophilic and non-toxic. Earlier, the biopolymer film was fabricated by using alginate 1\% wt and chitosan 1\% wt. solutions at volume ratios of 99:1 and 97:3. Next, the biopolymer film solution was cross-linked with $1 \mathrm{M} \mathrm{CaCl}_{2} .2 \mathrm{H}_{2} \mathrm{O}$ for two hours and later dried for 24 hours at room temperature. Then, the surface properties of the prepared biopolymer films were characterised via Field Emission Scanning Electron Microscopy (FESEM), Atomic Force Microscopy (AFM) and contact angle measurement. It was observed that the surface of the biopolymer film became rougher as the volume of the chitosan increases. This condition was confirmed with average surface roughness, $R_{A}$ for biopolymer film with ratio of 97:3 resulted in higher values. Also, it was found that the surface of biopolymer films were hydrophilic after the contact angle was less than $90^{\circ}$. This can be concluded that the biopolymer based on alginate/chitosan is a promising candidate for wound healing materials particularly with good surface properties for faster healing process at the wound areas.
\end{abstract}

\section{Introduction}

A skin wound is a disruption of skin structure and function usually due to, among others, acute burns or chronic ulcers. Normally, wound healing materials are used to cure the area of affected skin. The main aim of wound healing is to have a faster curing period with elimination of antibacterial infection [1]. Currently, film materials or dressings based on polymers are one of the most popular choices as a wound healing material. Film dressings are simple, thin and permit the exchange of water vapour and oxygen at the curing site [2].

Alginate is a natural polymer based of hydrogels from the anionic polysaccharide group. It has very good biocompatibility and hydrophilicity properties. Alginate film exhibits a moist environment which promotes the healing process and facilitates easy removal. Also, it has a higher tissue compatibility, low toxicity and good mucoadhesive properties. This led the selection of alginate as a biomaterial for wound healing materials [3-4]. On the other hand, chitosan is chosen as a wound healing material due to its biocompatibility, biodegradability, haemostatic activity, antiinflectional activity, and also able to accelerate the wound healing process. The major advantage of chitosan is due to its antibacterial properties as well as lower toxicity towards mammalian cells [5].

Therefore, natural biopolymers comprised of alginate and chitosan were fabricated in order to obtain the combined outstanding properties of the wound healing materials (Fig. 1). Several characterizations were also carried out and presented in this study. 


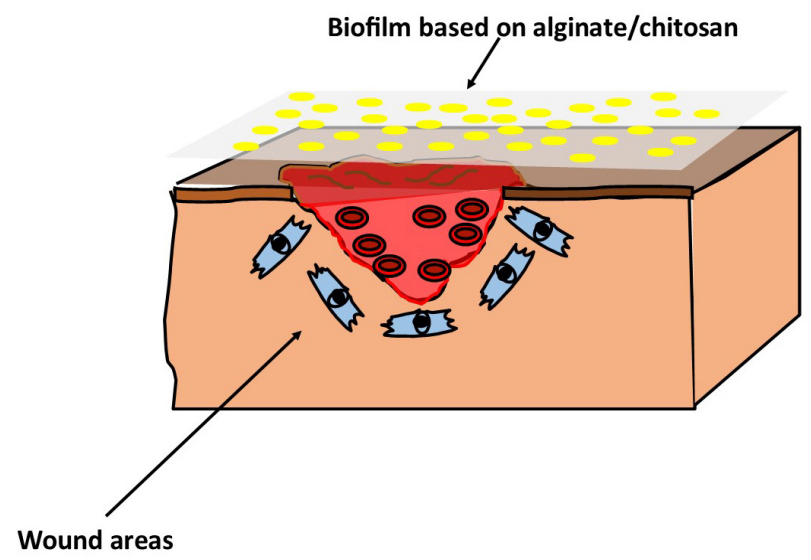

Fig. 1: Schematic diagram of biopolymer based on alginate/chitosan applied at the wound areas

\section{Materials and Methods}

The raw materials used were sodium alginate, chitosan from shrimp shells (practical grade) and calcium chloride dehydrate $\left(\mathrm{CaCl}_{2} \cdot 2 \mathrm{H}_{2} \mathrm{O}\right)$ that were purchased from Sigma-Aldrich. Basically, the preparation of the biopolymer alginate/chitosan film using solution casting method which is comprised of three main stages (Fig. 2). The first stage involved the preparation of alginate $1 \mathrm{wt} . \%$ and chitosan $1 \mathrm{wt} . \%$ solutions. Then, the second stage was the casting of the biopolymer film at volume ratios of alginate to chitosan solution which were 99:1 and 97:3. These solutions were then cross-linked with $1 \mathrm{M} \mathrm{CaCl}_{2} \cdot 2 \mathrm{H}_{2} \mathrm{O}$ for two hours. The last stage was drying of the alginate/chitosan film at room temperature for a duration of 24 hours.

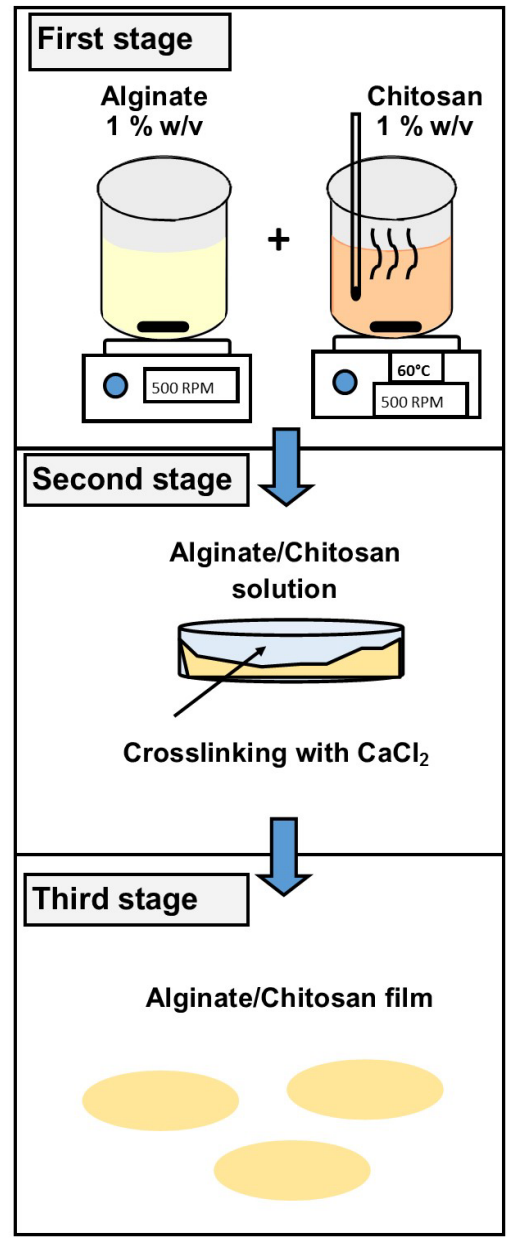

Fig. 2: The fabrication stages of biopolymer alginate/chitosan film 
All the fabricated biopolymer films at the different ratios were observed with Field Emission Scanning Electron Microscopy (FESEM) (JEOL, Japan) in order to observe the surface morphology of the films. The surface roughness of biofilms were measured by using the Atomic Force Microscopy (AFM) (XE-100, Republic of Korea). The contact angle was conducted with RaméHart (Model $200 \mathrm{~F} 1230 \mathrm{~V}$ ) to measure the contact angle of a water droplet on the biopolymer film surfaces in accordance with ASTM D7490.

\section{Results and Discussion}

Microstructure Observation. The micrographs of the biopolymer films at the different ratios are presented in Fig. 3. It can be seen Fig. 3 (a) that the alginate/chitosan biofilm at the ratio of 99:1 has less pores as compared with Fig. 3 (b). This may be due to the higher content of chitosan that affected the surface morphology of the film. It was known that chitosan is polycationic polymers and has positive charges which normally cross-linked with chemical agents such as glutaraldehyde, formaldehyde and genipin [6]. Therefore, it seems like alginate coagulated with chitosan in order to form a solid film.

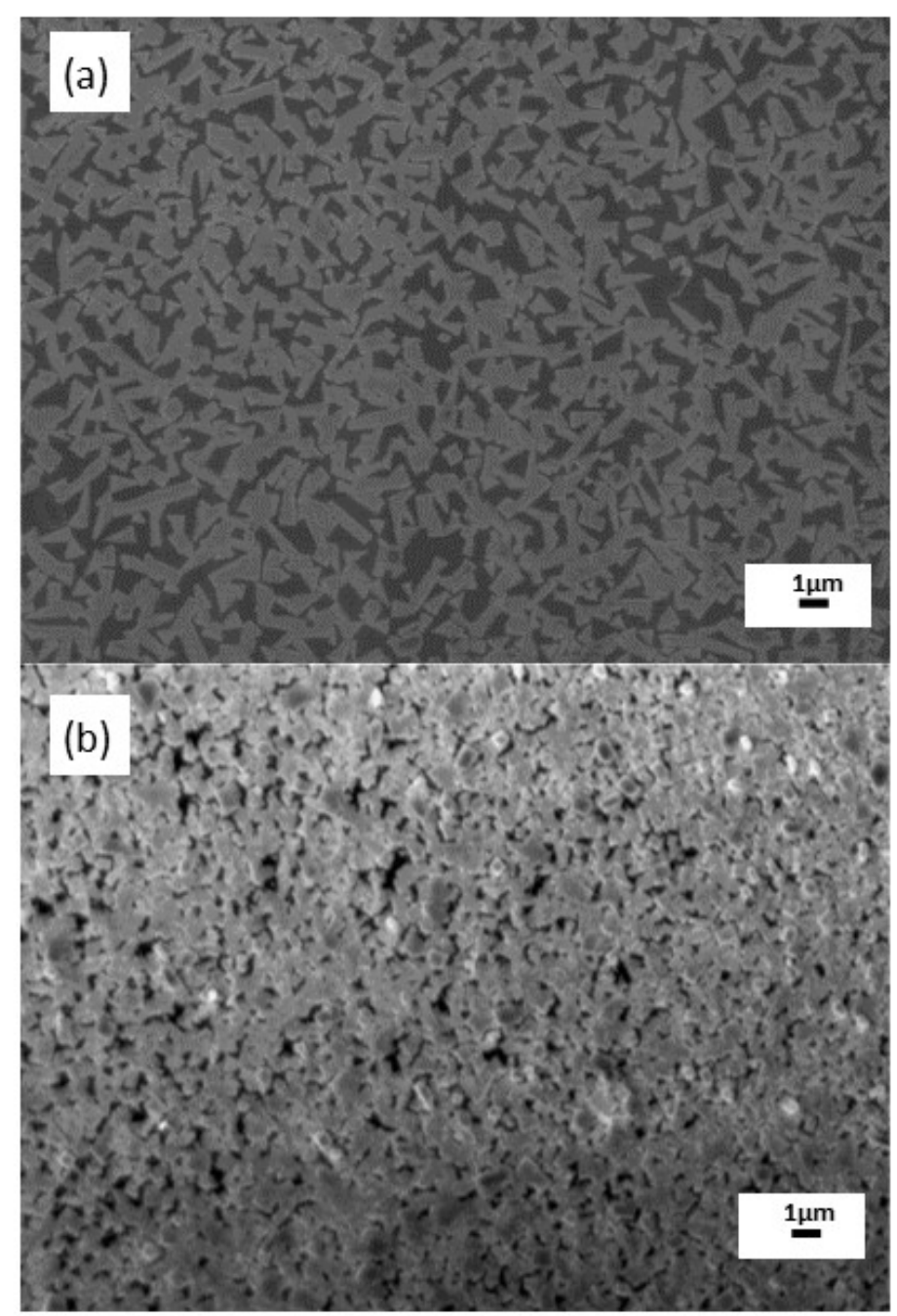

Fig.3. FESEM images of the biopolymer alginate/chitosan films at different ratios of (a) 99:1 and (b) $97: 3$

Surface Roughness Measurement. The surface topography of the biopolymer films are presented in Fig. 4. It was found that the average surface roughness, $\mathrm{R}_{\mathrm{A}}$ of the alginate/chitosan biofilms at ratios of $99: 1$ and 97:3 were $4.6 \pm 1.24 \mathrm{~nm}$ and 7.0 $\pm 2.94 \mathrm{~nm}$, respectively. This confirms the surface roughness of the biopolymer film increases as the volume of chitosan increased. 


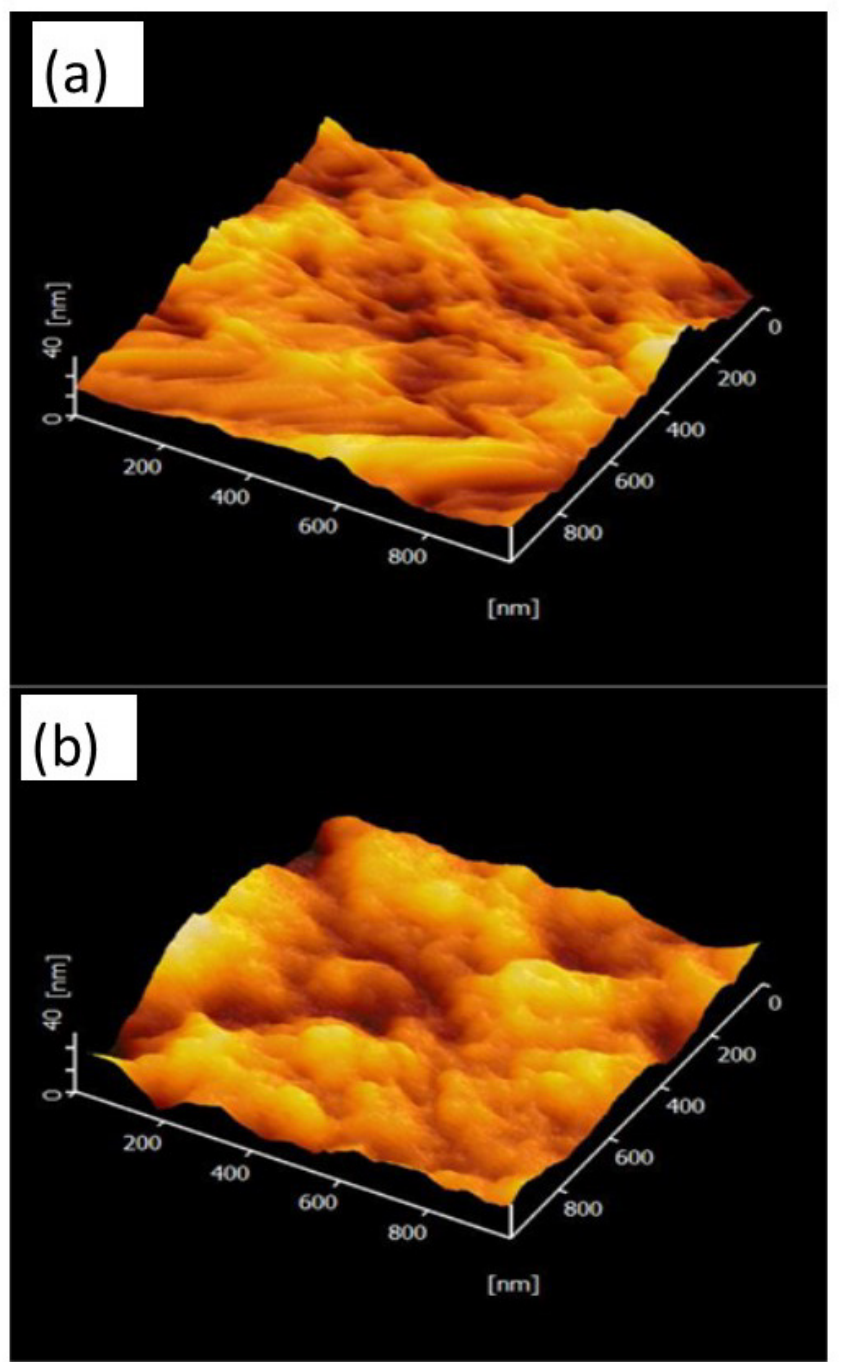

Fig.4: AFM images of biopolymer alginate/chitosan film at different ratios of (a) 99:1 and (b) 97:3

Contact Angle Measurement. One of the most important surface properties of wound material is their surface wettability. The wettability depends on the degree of the contact and interaction between the material and the biological or wound environment. Fig. 5 shows that the contact angle of biopolymer alginate/chitosan film at ratios of $99: 1$ and $97: 3$ were $24.70^{\circ}$ and $21.90^{\circ}$, respectively. This exhibits the fabricated biopolymer alginate/chitosan films have hydrophilicity properties (less than $90^{\circ}$ ) which help to accelerate the recovery process. 

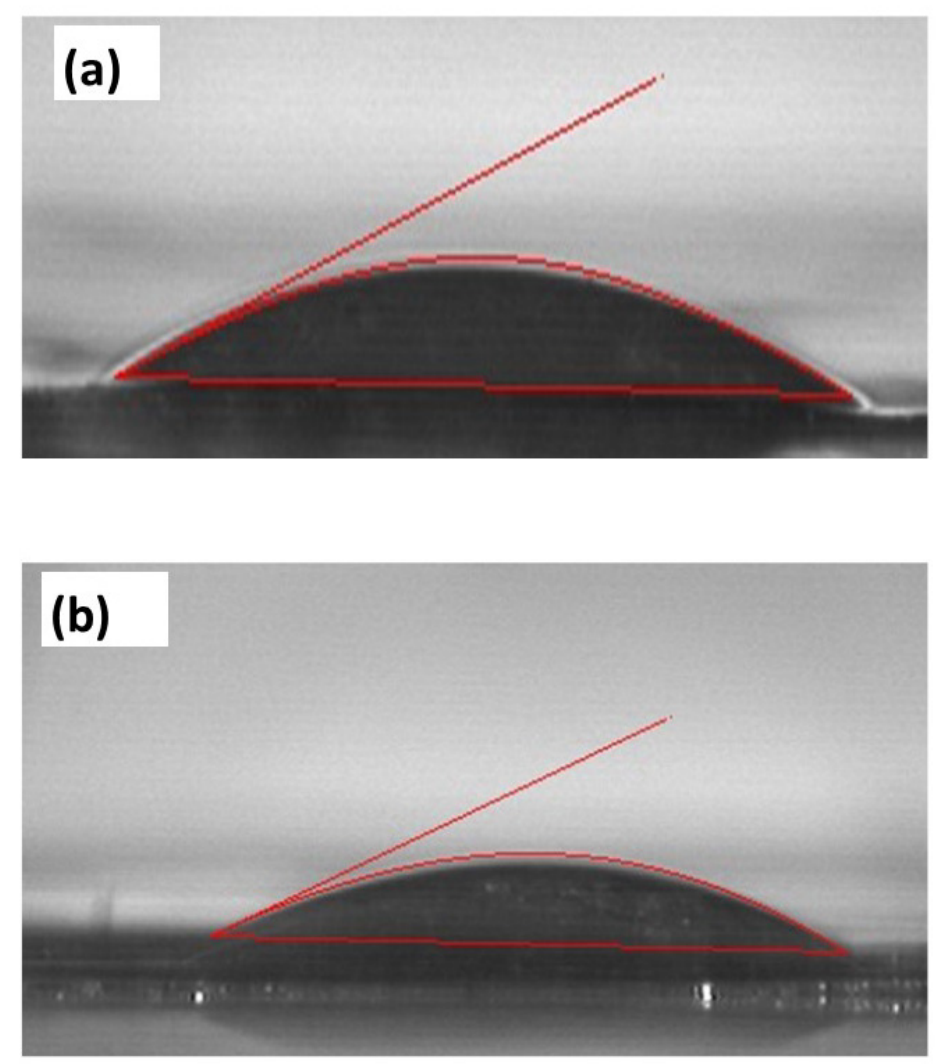

Fig. 5: Contact angle images of biopolymer alginate/chitosan film at different ratios of (a) 99:1 and (b) $97: 3$

\section{Summary}

Natural biopolymer films based on alginate/chitosan at different volume ratios were successfully fabricated via the solution casting method. It was revealed that the surface properties of the biopolymer film increased with chitosan. This indicates the rougher surfaces of the film will give a good adhesion when in contact at the wound areas during the healing process. Also, the hydrophilicity properties were obtained from the biopolymer films which allows for a faster healing process.

\section{Acknowledgement}

The authors would like to acknowledge the Ministry of Education Malaysia and Universiti Tun Hussein Onn Malaysian for granting the FRGS (K063) and TIER 1 (H188) grants which enabled the undertaking of this study.

\section{References}

[1] S. Das, A. B. Baker, Biomaterials and nanotherapeutics for enhancing skin wound healing. Frontiers in bioengineering and biotechnology, 4 (2016) 82.

[2] M. Rezvanian, M.C.I.M Amin, S.F. Ng, Development and physicochemical characterization of alginate composite film loaded with simvastatin as a potential wound dressing. Carbohydrate polymers, 137 (2016) 295-304

[3] J. Boateng, R. Burgos-Amador O. Okeke, H. Pawar, Composite alginate and gelatin based biopolymeric wafers containing silver sulfadiazine for wound healing. International journal of biological macromolecules, 79 (2015) 63-71. 
[4] B. Cohen, O. Pinkas, M. Foox, M. Zilberman, Gelatin/alginate novel tissue adhesives and their formulation ${ }_{i}$ strength effects. Acta biomaterialia, 9(11) (2013) 9004-9011

[5] D. Archana, J. Dutta, PK Dutta, Evaluation of chitosan nano dressing for wound healing: Characterization, invitro and in vivo studies. International journal of biological macromolecules, 57 (2013) 193-203.

[6] V.R. Sinha, A. K. Singla, S. Wadhawan, R. Kaushik, R. Kumria, K, Bansal, S. Dhawan, Chitosan microspheres as a potential carrier for drugs. International Journal of Pharmaceutics 274 (2004) 1-33 